\title{
High-Resolution Distributed Differential Curvature Measurement Based on Phase-Sensitive Optical Time Domain Reflectometry and Multi-Core Fiber
}

\author{
Lukasz Szostkiewicz ${ }^{1,3,4}$, Zhisheng Yang ${ }^{3}$, Marcelo A. Soto ${ }^{3, \dagger}$, Alejandro Dominguez-Lopez ${ }^{2}$, Anna Pytel ${ }^{1,3}$, \\ Agnieszka Kolakowska ${ }^{2,3}$, Michalina Jóźwik ${ }^{2,3}$, Krzysztof Markiewicz², Marek Napierala², \\ Luc Thévenaz ${ }^{3}$, Tomasz Nasilowski² \\ ${ }^{1}$ Polish Centre For Photonics And Fibre Optics, 312 Rogoznica, 36-060 Glogow Malopolski, Poland \\ ${ }^{2}$ InPhoTech Sp. z o. o., 12 Dzika st 15 Warsaw, 00-195, Poland \\ ${ }^{3}$ EPFL Swiss Federal Institute of Technology, Institute of Electrical Engineering, SCI STI LT, Station 11, CH-1015 Lausanne, Switzerland \\ ${ }^{4}$ Faculty of Physics, Warsaw University of Technology, Warsaw 00-662, Poland \\ ${ }^{\dagger}$ Current address: Department of Electronic Engineering, Universidad Técnica Federico Santa María, 2390123 Valparaiso, Chile \\ Authore-mail address: lszostkiewicz@pcfs.org.pl
}

\begin{abstract}
A distributed curvature sensor based on multicore fiber and phase-sensitive optical time domain reflectometry is presented, demonstrating a resolution of $10 \mathrm{~cm}$ over $\sim 25 \mathrm{~m}$ of sensing range.(C) 2018 The Author(s)

OCIS codes: (290.5870 Scattering, Rayleigh; (060.2370) Fiber optics sensors;
\end{abstract}

\section{Introduction}

In the last few decades, distributed optical fiber sensors have settled as the most convenient approach to continuously monitor long distances of fiber, featuring a vast amount of sensing points. These sensors are based on the analysis of the scattered light within the optical fiber, due to either Rayleigh, Brillouin or Raman effects [1]. The vast majority of distributed optical fiber sensors are dedicated to strain or temperature measurements with ever increasing sensing resolution and reduced measurement time [2]. Concentration on strain and temperature is due to the ITU G.652 fiber relative insensitivity to other physical quantities. On the other hand, recently, the development of specialty optical fibers like few modes [3], multi-core [4] or hollow-core [5] enabled to perform measurements that are not possible with single-mode fibers like pressure sensing [6], axial stress sensing [7] or gas detection [8]. One of the most promising approach is to detect the trajectory of the fiber (shape) using the analysis of differential strain distribution along the multiple cores of a fiber. The first demonstration of such a sensor based on multi-core fibers is presented in [9], where Brillouin Optical Time Domain Analysis (BOTDA) was used to extract the fiber shape.

In this paper a novel highly-sensitive distributed shape sensor is proposed based on phase-sensitive Optical Time Domain Reflectometry ( $\phi$-OTDR) [10]. Making use of a multi-core fiber and the coherent Rayleigh scattering measured along different fiber cores, displacement measurements with extremely high precision and high spatial resolution are experimentally demonstrated.

\section{Sensing principle}

By measuring simultaneously the strain deviations of several cores within a multi-core optical fiber, whose geometry is known, it is possible to extract the information about the bending and/or curvature at each section of the fiber. Fig. 1 depicts the scheme of the MCF structure at the reference position, where all of the cores (cores $\mathrm{C} 1, \mathrm{C} 2, \mathrm{C} 3$ ) lay on the $\mathrm{XY}$ plane, and their position when a certain torsion is applied to the fiber (cores P0, P1, P2, P3), where:

$$
\begin{aligned}
& P 1=\left(d \cos \theta_{1}, d \sin \theta_{1}, \varepsilon_{1}\right), \\
& P 2=\left(d \cos \theta_{2}, d \sin \theta_{2}, \varepsilon_{2}\right), \\
& P 3=\left(d \cos \theta_{3}, d \sin \theta_{3}, \varepsilon_{3}\right)
\end{aligned}
$$

The coordinates defining the position of the central core are $P 0=\left(0,0, \varepsilon_{\text {const. }}\right)$, where $\varepsilon_{\text {const. }}$ is the strain experienced by all of the cores of the MCF. In the same way, the location of a point $P$ is defined as $P=\left(d \cos \theta_{b}, d \sin \theta_{b}, \varepsilon_{b}+\varepsilon_{\text {const. }}\right)$. The bend orientation is given by $\theta_{b}$, while $\varepsilon_{b}=d k$ is the strain induced by such bending, where $k=1 / R$ is the curvature associated to a given radius of curvature $R$. To calculate the position of point $P$, a normal vector $\vec{n}=\left(n_{x}, n_{y}, n_{z}\right)$ is defined from the plane which $P 1, P 2$, and $P 3$ form, verifying that $\vec{n}=(P 2-P 1) \times(P 3-P 1)$. Then, based on the $x$ and $y$ coordinates of the normal vector $\vec{n}$, the bending orientation is defined as: 


$$
\theta_{b}=\arctan \frac{n_{y}}{n_{x}}
$$

Since the $x$ and $y$ coordinates of point $P$ are known (due to the known structure of the fiber), as well as the plane in which it is lying (given by the normal vector $\vec{n}$ ), by knowing the bending orientation, one can exactly locate the position of point $P$. Hence, one can define the equation of the plane for this point, as:

$$
0=n_{x} d \cos \theta_{b}+\mathrm{n}_{y} d \sin \theta_{b}+\mathrm{n}_{z}\left(\varepsilon_{b}+\varepsilon_{\text {const. }}\right)+\varepsilon_{\text {const }}
$$

Then, solving for the only unknown, the strain induced by bending can be written as:

$$
\varepsilon_{b}=-\frac{n_{x}}{\mathrm{n}_{z}} d \cos \theta_{b}-\frac{\mathrm{n}_{y}}{\mathrm{n}_{z}} d \sin \theta_{b}-\frac{\varepsilon_{\text {const. }}}{\mathrm{n}_{z}}-\varepsilon_{\text {const. }}
$$

Thus, the curvature is equal to:

$$
k=\left|\varepsilon_{b} / d\right|
$$

Consequently, if the strain $\left(\varepsilon_{b}\right)$ is known, one can extract the bending orientation $\left(\theta_{b}\right)$ and the curvature $(k)$ at each point along the fiber. Such a methodology is independent of the initial choice of the cores. Any 3 cores with different position that are chosen will be sufficient to perform the analysis.
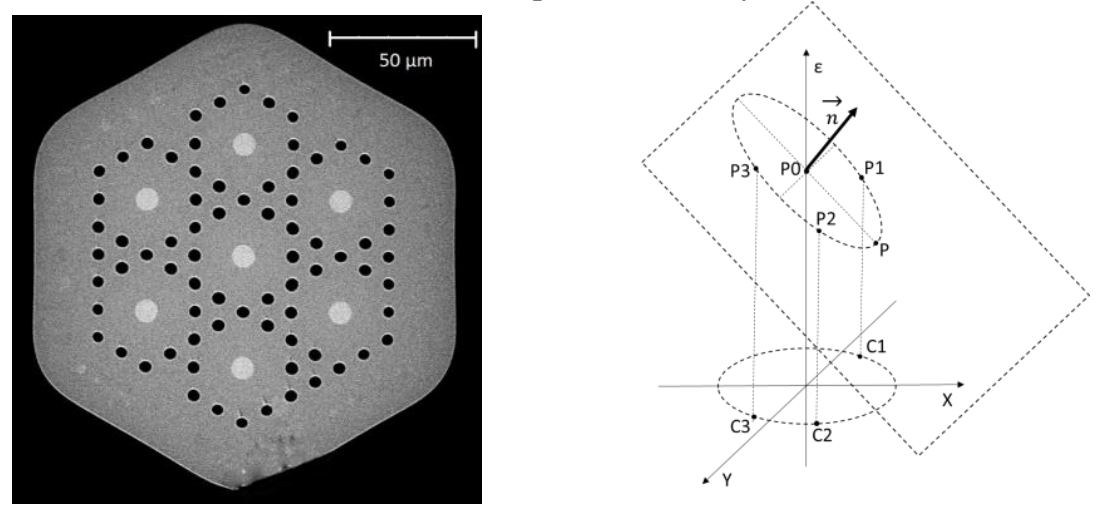

Fig. 1. a) Cross-section of the seven-core fiber used in the experiment. b) Analytical drawing of the MCF subject to a non-homogeneous strain along all of its cores (i.e. bending). At the bottom, all of the cores are aligned with the plane XY at the origin. At the top, the cores are displaced and within a plane tilted by a certain angle with respect to the reference position.

\section{Experimental Setup}

In order to study the above-mentioned approach, the scheme presented in Fig. 2 has been set up, which is a highperformance implementation of a $\phi$-OTDR sensor. In this case, the light source is a Distributed-Feedback (DFB) laser, whose wavelength was directly swept by tuning the bias current of the laser. Afterwards, the pulse is shaped by means of an Electro-Optical Intensity Modulator (Mach-Zehnder modulator) which allows producing sharp and short pulses of $\sim 1 \mathrm{~ns}$ width, corresponding to a spatial resolution of $10 \mathrm{~cm}$. The pulse width is externally driven by an electrical pulse generator. However, to ensure a sufficiently high Extinction Ratio (ER) of the optical pulses, a second EOM was used to gate the pulses, and hence, further reduce the out-of-pulse Rayleigh backscattered power (which provides no local information and eventually distorts the measurements). Aiming at increasing the pulse peak power delivered to the fiber, an Erbium-Doped Fiber Amplifier (EDFA) is inserted. The amplifier stage is followed by a Variable Optical Attenuator (VOA) to accurately select the desired power in order to avoid non-linear effects in the fiber [6]. After going through the circulator and prior to entering the fiber, an optical switch is used to be able to ensure comparable measurements of all the cores of interest in an automatized manner. The outputs of the optical switches are connected to the Fan-in/Fan-out device that couples the light from seven SMF to each core of the MCF, which features a maximum Insertion Loss (IL) of $\sim 1.1 \mathrm{~dB}$ per core. The backscattered light coming out of port three of the circulator is again amplified by means of an EDFA to ensure a sufficiently high power upon the detector. In this case, a tunable Band-Pass Optical Filter (BPOF) selects the signal of interest, filtering out all of the Amplified Spontaneous Emission (ASE) coming out of the EDFA, and thus, limiting the sources of noise in the photodetector [7]. This photodetector features a bandwidth of $3 \mathrm{GHz}$, which is high enough to effectively grant a spatial resolution of 10 $\mathrm{cm}$. Ultimately, the electrical signal coming from the detector is acquired and processed by means of a highbandwidth oscilloscope. 


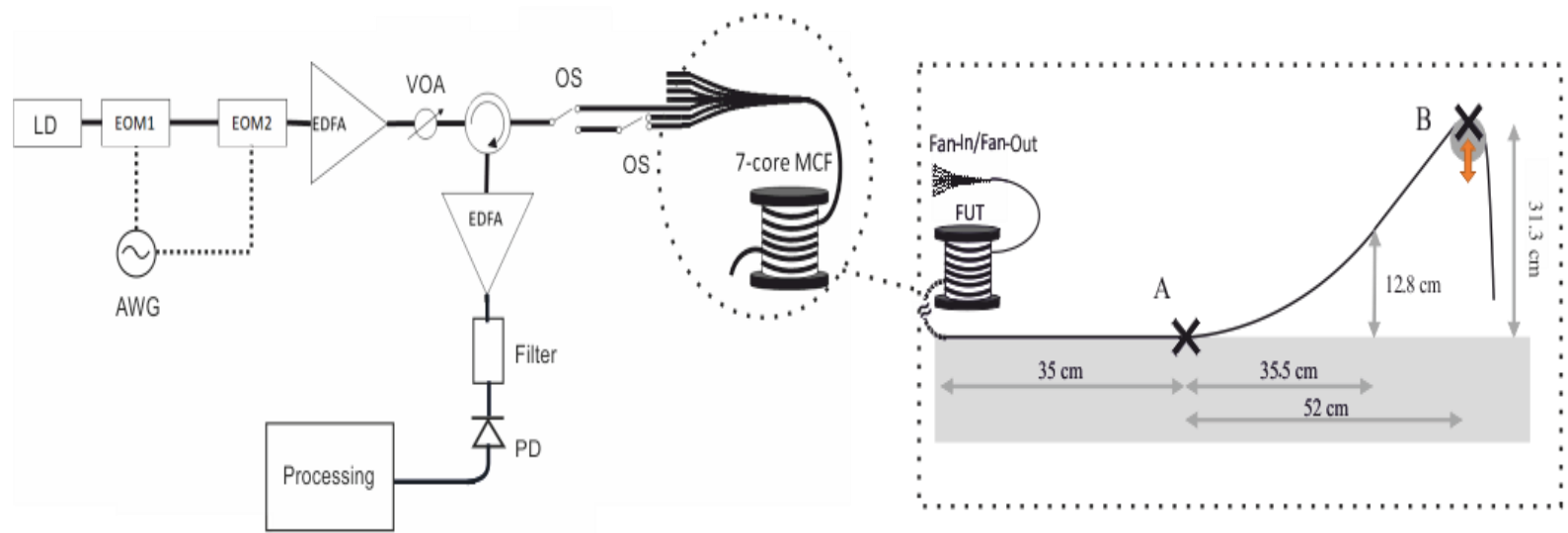

Fig. 2. Phase-sensitive OTDR experimental setup. EOM: Electro-Optical Modulator, EDFA: Erbium-Doped Fiber Amplifier; VOA: Variable Optical Attenuator; MCF: Multi-Core Fiber; FAN-IN/FAN-OUT. INSET: Displacement setup. The MCF is attached to a vertical-moving stage on its further end (point B), and $\sim 60 \mathrm{~cm}$ of fiber are suspended on air until reaching the base at point $\mathrm{A}$, where it is fixed.

Aiming at testing the capabilities of the presented scheme, an extra setup has been devised. The right-hand side of Fig. 2 shows the last section of the 24 m-long FUT, in which the fiber has been attached to a computercontrolled translation stage (point B in Fig. 1), elevated $31.3 \mathrm{~cm}$ from the base. Due to the high sensitivity of the system, the moving platform is set to shift its position upwards or downwards in steps of $\pm 2 \mathrm{~mm}$, with a total displacement range of $2 \mathrm{~cm}$. To secure comparable measurements, the MCF is also fixed to the base, as it is illustrated in point A of Fig. 2, which limits the length of the moving section of the fiber to be $\sim 60 \mathrm{~cm}$ long.

\section{Experimental results}

Making use of the aforementioned setup, a series of frequency sweeps were performed while varying the elevation of the fiber attached to the moving-stage in steps of $2 \mathrm{~mm}$. The DFB frequency was swept in steps of $50 \mathrm{MHz}$ (by acting on the laser bias current), until reaching a total range of $13.5 \mathrm{GHz}$, which brings a minimum strain sensitivity of $\pm 331 n \varepsilon$ and a full dynamic range of $\square 89 \mu \varepsilon$, according to Eq. (4). Fig. 3(a) illustrates the accumulated strain along the fiber for each elevation step of the moving-stage (point B in Fig. 2), where the strain has been estimated as the summation of the computed strain for each pair of vertical positions for three cores of the fiber. The evolution of the accumulated strain is given when the moving-plate is elevating the fiber $+20 \mathrm{~mm}$. The cumulated strain for the three cores is null before reaching $23 \mathrm{~m}$, which is in accordance with the point where the fiber is attached to the base (point A in Fig. 2). After that, and up to $23.16 \mathrm{~m}$, the second core (in red) experiences a positive strain (elongation), meanwhile the fourth and fifth cores (blue and green, respectively) experience a negative strain (compression). At that point in the fiber, there is a change in curvature, and thus, there is no strain induced at any of the cores. Subsequently, from $\sim 23.16 \mathrm{~m}$ to $23.6 \mathrm{~m}$, the fourth and fifth cores (blue and green) experience a positive strain (elongation) whilst the second core (red) this time suffers a negative strain or compression. A symmetrical behavior of cores 2 (red) and 4 (blue) is easily observed, from which it can be inferred their opposite location within the geometry of the MCF.

It should be highlighted that the issue of strain-temperature cross-sensitivity can be easily overcome in this scheme, since the contribution of temperature to the frequency correlation peak-shift has the same influence on all the cores of the MCF, and hence, it can be easily detected and compensated. In addition, the section of fiber not subject to displacement can also be used to detect changes due to temperature variations, and it could be used as a reference. However, since the measurement is cumulative and because of the intrinsically-static acquisition time (each frequency scanning requires 270 steps), a cumulated error is expected towards the end of the set of measurements due to deterioration of the operation parameters, such as pulse ER, and/or nonlinearities in the slope applied to the laser bias current. 
a)

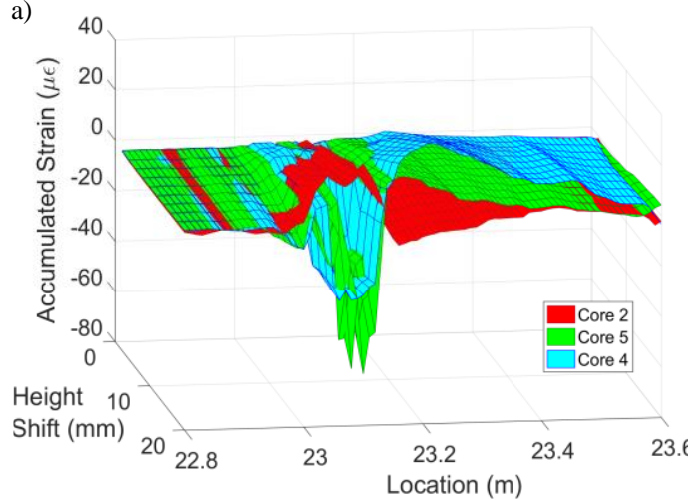

b)

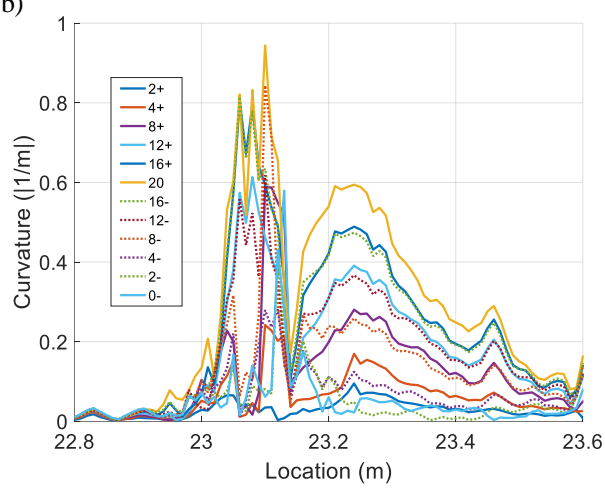

Fig. 3. a) Accumulated strain due to vertical displacement, estimated as the summation of the computed strain for each pair of vertical positions for three cores of the fiber, where the first reference sweep is done at the lowest point ( 0 mm of displacement). b) Measured curvature (absolute value) for a variable moving-stage height, starting at the bottom ( 0 mm elevation), shifting upwards up to 20 mm (continuous lines), and going down to the original position (dotted lines).

After obtaining the strain for each core, and making use of Eq. (5), one can compute the curvature along the entire fiber length. Fig. 3(b) illustrates the retrieved relative curvature change (in absolute value) for several heights of the moving-stage, starting at the lowest position (at $0 \mathrm{~mm}$ ), going upwards up to the highest location (at $20 \mathrm{~mm}$ ) and going downwards through the same steps towards the original position. The retrieved curvatures for each step show a strong repeatability, though, as mentioned before, the measured steps obtained after a longer time are affected by a higher accumulated error.

\section{Conclusion}

In conclusion, we have presented, to the best of our knowledge, the first distributed curvature/bending sensor based on multi-core fiber and phase-sensitive OTDR. The results demonstrate a high strain sensitivity (down to $\sim 0.3 \mu \varepsilon$ ) over a 24 m-long MCF with a spatial resolution of $10 \mathrm{~cm}$. By measuring the strain in only three lateral cores of the fiber one can compute the relative curvature change at each point and retrieve the shape of the fiber. The proposed scheme avoids the commonly faced issues of cross-sensitivity between strain and temperature, due to its differential working principle. Although the proof of concept is experimentally validated, the issue of long acquisition times must be addressed in order to facilitate the practical implementation of this approach. The here presented results illustrate the capabilities of the use of specialty optical fibers for distributed sensing, establishing the foundations of a broad range of new applications, such as dynamic shape sensing.

Acknowledgements: The project leading to this application has received funding within the „NODUS” project carried out within the TEAMTECH programme of the Foundation for Polish Science co-financed by the European Union under the European Regional Development Fund

\section{References}

[1] X. Bao and L. Chen, "Recent Progress in Distributed Fiber Optic Sensors," Sensors 12(12), 8601-8639 (2012).

[2] Zhisheng Yang, Marcelo A. Soto, Desmond M. Chow, Pabitro Ray, and Luc Thévenaz, "Brillouin Distributed Optical Fiber Sensor Based on a Closed-Loop Configuration," J. Lightwave Technol. 36, 1239-1248 (2018)

[3] An Li, Yifei Wang, Qian Hu, and William Shieh, "Few-mode fiber based optical sensors," Opt. Express 23, 1139-1150 (2015)

[4] A. Ziolowicz, et al., "Hole-assisted multicore optical fiber for next generation telecom transmission systems", Appl. Phys. Lett. 105, 081106 (2014).

[5] Yuechuan Lin, Wei Jin, Fan Yang, Yanzhen Tan, and Hoi Lut Ho, "Performance optimization of hollow-core fiber photothermal gas sensors," Opt. Lett. 42, 4712-4715 (2017)

[6] Jun Ma, Wei Jin, Hoi Lut Ho, and Ji Yan Dai, "High-sensitivity fiber-tip pressure sensor with graphene diaphragm," Opt. Lett. 37, 2493$2495(2012)$

[7] De Pauw, B., Goossens, S., Geernaert, T., Habas, D., Thienpont, H., \& Berghmans, F. (2017). Fibre Bragg Gratings in Embedded Microstructured Optical Fibres Allow Distinguishing between Symmetric and Anti-Symmetric Lamb Waves in Carbon Fibre Reinforced Composites. Sensors, 17(9), 1948.

[8] Yuechuan Lin, Fei Liu, Xiangge He, Wei Jin, Min Zhang, Fan Yang, Hoi Lut Ho, Yanzhen Tan, and Lijuan Gu, "Distributed gas sensing with optical fibre photothermal interferometry," Opt. Express 25, 31568-31585 (2017)

[9] Z. Zhao, et al., "Distributed shape sensing using Brillouin scattering in multi-core fibers," Opt. Express 24(22), 25211 (2016).

[10] Y. Koyamada, et al., "Fiber-Optic Distributed Strain and Temperature Sensing With Very High Measurand Resolution Over Long Range Using Coherent OTDR," J. Light. Technol. 27(9), 1142-1146 (2009). 